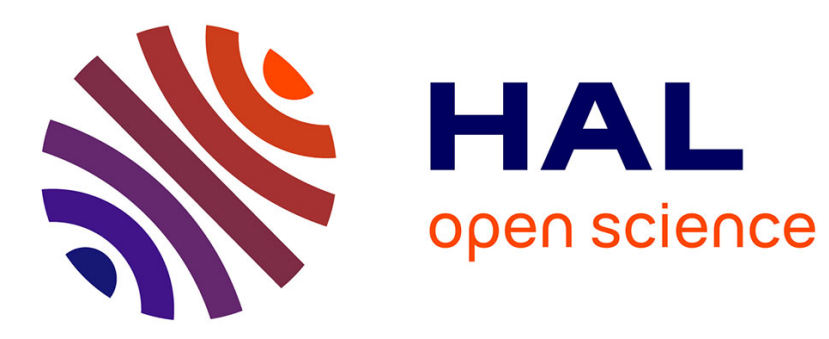

\title{
Cascaded model-free control for trajectory tracking of quadrotors
}

\author{
Maria Bekcheva, Cédric Join, Hugues Mounier
}

\section{To cite this version:}

Maria Bekcheva, Cédric Join, Hugues Mounier. Cascaded model-free control for trajectory tracking of quadrotors. International Conference on Unmanned Aircraft Systems, ICUAS'18, Jun 2018, Dallas, TX, United States. 10.1109/icuas.2018.8453339 . hal-01831468

\section{HAL Id: hal-01831468 \\ https://hal.science/hal-01831468}

Submitted on 24 Jul 2020

HAL is a multi-disciplinary open access archive for the deposit and dissemination of scientific research documents, whether they are published or not. The documents may come from teaching and research institutions in France or abroad, or from public or private research centers.
L'archive ouverte pluridisciplinaire HAL, est destinée au dépôt et à la diffusion de documents scientifiques de niveau recherche, publiés ou non, émanant des établissements d'enseignement et de recherche français ou étrangers, des laboratoires publics ou privés. 


\title{
Cascaded Model-Free Control for trajectory tracking of quadrotors
}

\author{
Maria Bekcheva $^{1}$, Cédric Join ${ }^{2}$ and Hugues Mounier ${ }^{1}$
}

\begin{abstract}
In the subject of quadrotor controller design, usually modelling and identification are tedious and timeconsuming tasks. In this study, we propose a controller design that avoids the quadrotor's system identification procedures while staying robust with respect to endogenous and exogenous disturbances. To reach our goal, based on the cascaded structure of a quadrotor, we divide the system into positional and attitude subsystems each controlled by an independent model-free controller. We validate our control approach in two realistic scenarios : in presence of unknown measurement noise and unknown time-varying wind disturbances. We provide simulations on a realistic nonlinear quadrotor model following an aggressive position-yaw trajectory.
\end{abstract}

\section{INTRODUCTION}

In the last decade the use of unmanned aerial vehicles (UAVs) has increased significantly. From package delivery services, military uses and disaster management to photography and entertainment, the use-cases are numerous. Among the UAVs, quadrotors have been subject to intense research and development. One of the main challenges is modeling quadrotor dynamics and parameters estimation. This process can be tedious, time consuming and prone to modeling errors. A considerable amount of the literature has been devoted to model-based controls (Backstepping and sliding-mode techniques [4], Inner-Outer Loop Control by applying PID controller for the inner-loop and nested saturation controller for the outer-loop [5], Generalized Proportional Integral (GPI) observer based controller [19], Model Predictive Control [2]) among other methods. In these model-based approaches, the modeling accuracy can directly impact the controller success and performance. Moreover, many quadrotor applications are outdoor and may be faced with environmental uncertainties. Among the meteorological uncertainties, the wind effect has one of the highest impacts on the quadrotor performance while being very hard to predict. Therefore, in the model definition, the wind is usually considered as a predefined constant. As suggested in the papers [3], [17], [14], in practice, using a PID have shown more advantages than more advanced quadrotor controllers because of its simplicity and robustness. We chose to use the model-free control (MFC) since it has been already shown successful in practice (see [9], [6] and the references therein, and [1], [15], [16]) that the MFC have better performances

\footnotetext{
${ }^{1}$ Maria Bekcheva and Hugues Mounier are with the Laboratoire de Signaux et Systèmes (L2S, UMR CNRS 8506), CNRS, CentraleSupélec, Université Paris-Sud, France (e-mail: maria.bekcheva@12s.centralesupelec.fr, hugues.mounier@12s.centralesupelec.fr

${ }^{2}$ Cédric Join is with CRAN (CNRS, UMR 7039), Université de Lorraine, BP 239, 54506 Vandœvre-lès-Nancy, France, A.L.I.E.N. (ALgèbre pour Identication \& Estimation Numériques) and Projet Non-A, INRIA Lille Nord-Europe, France (e-mail: cedric.join@univ-lorraine.fr)
}

and advantages over a simple PID. These facts motivate us to present a cascaded-model-free approach for a quadrotor while considering more realistic situations like time-varying wind disturbances and measurement noise. We make our contribution in the following framework:

- We use a minimalist structure upon which our control scheme rests (see Equations (12), (14), (19a)-(19c)). This structure is independent of any mass, inertia, gyroscopic or aerodynamic effects; the only information we use is that each positional and rotational dynamics is of second order and that the thrust produced by the propellers is suitably projected onto the inertial frame.

- Because of the underactuated nature of the quadrotor, we consider an inner-outer structure of the quadrotor that allows us to divide the control into two stages. At the first stage, we show that it is possible to apply a model-free control in the outer loop and from there, we derive the desired thrust, and the desired roll and pitch angles. We then apply again the model-free control in the inner loop for the second stage.

- No precise information of the quadrotor physical parameters is required. For instance, it will be inconvenient and in some cases imprecise to measure the mass value [20] of the quadrotor together with its payload during each flight.

- For the simulations, we have tested our approach with a realistic quadrotor model that contains gyroscopic and aerodynamic effects.

Recent studies have shown the interest in dividing the control problem into two parts: one based on the model and the other based on the model-free control to cope with model uncertainties and/or external perturbations. In [21], modelfree sliding-mode control (based on a linearized model) is studied, in [23] a model-free backstepping control (based on a linearized model) and an LQR control have been experimentally tested on a real system. In these studies, the control law is still partially linked to the system model knowledge.

In several works, the yaw angle is assumed to be zero due to high nonlinear coupling in position and attitude dynamics. In [22], an event-driven model-free control is compared with several other controls while taking zero reference for the yaw angle. This limits the maneuverability of quadrotors. In our case we show that the quadrotor can follow aggressive maneuvers while tracking the yaw angle $\psi$. This is a huge advantage with clear benefits on time and energy. The positionyaw tracking as mentioned in [18] can be useful in different use-cases e.g. Aerial Screwdriver task (the quadrotor should 


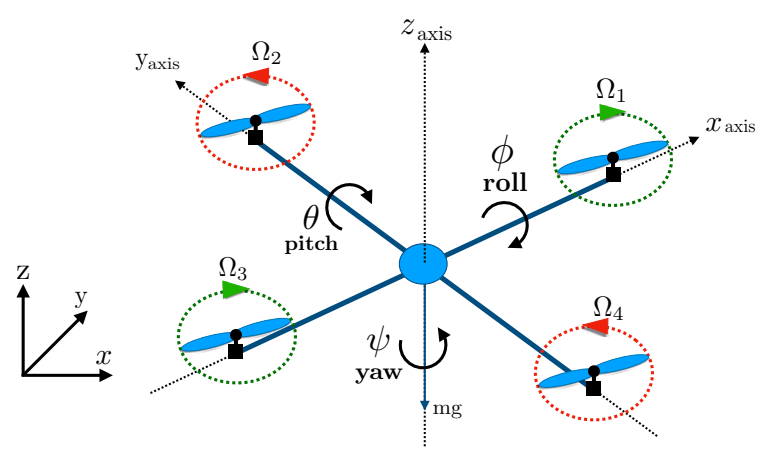

Fig. 1. The quadrotor system.

turn a screw), the Aerial Grasp (multiple quadrotors rotate their $\psi$-angle to grasp an object), etc.

This paper has the following outline. In Section II, we briefly describe the quadrotor dynamics focusing on its structure. Then in Section III, we present our cascaded model-free approach. Finally, we validate our approach in Section V by testing it on two scenarios when the quadrotor is faced with unknown measurement noise and with unknown timevarying wind disturbances. The aggressive position trajectory is a B-spline curve with predefined control points.

\section{QUADROTOR MODEL}

The quadrotor is an underactuated system because it has six degrees of freedom but only four actual inputs. The six degrees of freedom include position motion in three directions and rotational motion around three axes. The schematic configuration of a quadrotor we adopted in this study is shown in Fig. 1 that includes the corresponding forces, angles and angular speeds.

A nonlinear model of quadrotor based on the NewtonEuler formalism (see the paper [11] for further information on the quadrotor model) is given by the following equations:

- Position dynamics

$$
\begin{aligned}
& m \ddot{x}=(\sin \psi \sin \phi+\cos \psi \sin \theta \cos \phi) u_{1}-A_{x} \dot{x}, \\
& m \ddot{y}=(-\cos \psi \sin \phi+\sin \psi \sin \theta \cos \phi) u_{1}-A_{y} \dot{y},
\end{aligned}
$$

$$
m \ddot{z}=-m g+\cos \theta \cos \phi u_{1}-A_{z} \dot{z},
$$

- Attitude dynamics

$$
\begin{aligned}
I_{x} \ddot{\phi} & =u_{2}+\dot{\theta} \dot{\psi}\left(I_{y}-I_{z}\right)+J_{r} \dot{\theta} \Omega_{r}, \\
I_{y} \ddot{\theta} & =u_{3}+\dot{\phi} \dot{\psi}\left(I_{z}-I_{x}\right)-J_{r} \dot{\phi} \Omega_{r}, \\
I_{z} \ddot{\psi} & =u_{4}+\dot{\theta} \dot{\phi}\left(I_{x}-I_{y}\right),
\end{aligned}
$$

where $x, y$ and $z$ are the position coordinates of the quadrotor's center of gravity, and $\theta, \phi$ and $\psi$ are the pitch, roll and yaw rotation angles respectively. The constant $m$ is the mass, $g$ is the gravitation acceleration, $I_{x}, I_{y}, I_{z}$ are the moments of inertia, and $J_{r}$ is the moment of inertia of the rotors. The controllers are: $u_{1}$ the total thrust generated by the four propellers applied in the $z$ direction; $u_{2}, u_{3}$ and $u_{4}$

\begin{tabular}{|l|c|}
\hline Symbols and values & Variables \\
\hline$m=0.53 \mathrm{~kg}$ & Quadrotor mass \\
$I_{x}, I_{y}=6.228 \times 10^{-3} \mathrm{~kg} \cdot \mathrm{m}^{2}$ & Inertia parameters \\
$I_{z}=1.121 \times 10^{-2} \mathrm{~kg} \cdot \mathrm{m}^{2}$ & Inertia parameter \\
$J_{r}=6.01 \times 10^{-5} \mathrm{~kg} \cdot \mathrm{m}^{2}$ & Rotor inertia moment \\
$b=3.13 \times 10^{-5} \mathrm{~N} \cdot \mathrm{s}^{2}$ & Thrust coefficient \\
$d=7.5 \times 10^{-7} \mathrm{Nm} \cdot \mathrm{s}^{2}$ & Drag coefficient \\
$A_{x}, A_{y}, A_{z}=0.25 \mathrm{~kg} / \mathrm{s}$ & Drag force coefficients \\
\hline \multicolumn{2}{|c|}{ TABLE I }
\end{tabular}

THE PARAMETERS AND THEIR CORRESPONDING VALUES FOR THE QUADROTOR SIMULATION.

the torques in the $\theta, \phi$ and $\psi$ directions respectively. The constants $A_{x}, A_{y}$ and $A_{z}$ are the drag force coefficients for velocities in the corresponding directions of the inertial frame.

The position dynamic model (1a)-(1c) contains the gravity force $m g$, the thrust force in the $z$ direction and the drag force. The attitude dynamic model (2a)-(2c) describing the roll, pitch and yaw rotations contains three terms which are the actuators action, the gyroscopic effect resulting from the rigid body rotation, and finally the gyroscopic effect resulting from the propeller rotation coupled with the body rotation. The control inputs $u_{1}, u_{2}, u_{3}, u_{4}$, and the speed $\Omega_{r}$ are defined as:

$$
\begin{aligned}
& u_{1}=b\left(\Omega_{1}^{2}+\Omega_{2}^{2}+\Omega_{3}^{2}+\Omega_{4}^{2}\right), \\
& u_{2}=b\left(\Omega_{4}^{2}-\Omega_{2}^{2}\right), \\
& u_{3}=b\left(\Omega_{3}^{2}-\Omega_{1}^{2}\right), \\
& u_{4}=d\left(\Omega_{1}^{2}+\Omega_{3}^{2}-\Omega_{2}^{2}-\Omega_{4}^{2}\right), \\
& \Omega_{r}=\Omega_{1}-\Omega_{2}+\Omega_{3}-\Omega_{4}
\end{aligned}
$$

where $\Omega_{i}$ is the angular speed of the $i$ th rotor, and $b$ and $d$ are the thrust and the drag coefficients respectively.

This model will be used for the simulation. Table I lists the parameter values used for the simulation model in Section V.

\section{Control Design}

In this section, first we summarize some of the main ideas on Model-Free Control (MFC) introduced in [9]. Then, we construct the cascaded-model-free controller for the quadrotor by using only a minimal nominal dynamics.

\section{A. Preliminaries for Model-Free Control}

The unknown differential equation describing the input/output behaviour of a finite-dimensional system with a single control variable $u$ and a single output variable $y$

$$
\begin{aligned}
& \mathbf{E}\left(y, \dot{y}, \ldots, y^{(\iota)}, u, \dot{u}, \ldots, u^{(\kappa)}\right)=0, \\
& \mathbf{E}: \mathbb{R}^{\iota+1} \times \mathbb{R}^{\kappa+1} \rightarrow \mathbb{R}, \mathbf{E} \in \mathbf{C}^{\infty}\left(\mathbb{R}^{\iota+1} \times \mathbb{R}^{\kappa+1}\right)
\end{aligned}
$$

can be described as: 
$y^{(\nu)}=\mathbf{E}\left(t, y, \dot{y}, \ldots, y^{(\nu-1)}, y^{(\nu+1)}, \ldots, y^{(\iota)} u, \dot{u}, \ldots, u^{(\kappa)}\right)$,

where $0<\nu \leqslant \iota, \frac{\partial E}{\partial y^{(\nu)}} \neq 0$. For simplicity, the Equation (4) can be represented in short time interval by an ultra-local model as:

$$
y^{(\nu)}=F+\alpha u
$$

where

- $\alpha \in \mathbb{R}$ is a non-physical constant parameter chosen such that $\alpha u$ and $y^{(\nu)}$ will be of the same order of magnitude. In the recent study [7], $\alpha$ is considered as a timevarying parameter in order to overcome the presence of unknown delays.

- the time-varying function $F(t)$ is approximated by a piecewise constant function. It represents the rest of the unmodelled dynamics in the input-output behaviour of the system and the unknown endogenous and exogenous disturbances. Therefore, it adapts to the changes of the system at each actuation step.

Obtaining a good estimate of $\hat{F}$ can be achieved by considering it as $\nu^{\text {th }}$ iterated integral on a short time interval $[t-T, t]$ (see [10]). For instance, when $\nu=2$, we can rewrite the equivalent of the equation (5) in the Laplace form as:

$$
s^{2} Y(s)-s y(0)-\dot{y}(0)=\frac{F}{s}+\alpha U(s)
$$

We get rid of the initial conditions $y(0)$ and $\dot{y}(0)$ by differentiating twice the equation (6) w.r.t. to the Laplace variable $s$.

$$
2 Y(s)+4 s \frac{d Y}{d s}+s^{2} \frac{d^{2} Y}{d s^{2}}=\frac{2 F}{s^{3}}+\alpha \frac{d^{2} U}{d s^{2}}
$$

To greatly attenuate the noise, we multiply the both sides of equation (7) by $s^{-3}$

$$
\frac{2}{s^{3}} Y(s)+4 \frac{1}{s^{2}} \frac{d Y}{d s}+\frac{1}{s} \frac{d^{2} Y}{d s^{2}}=\frac{2 F}{s^{6}}+\frac{\alpha}{s^{3}} \frac{d^{2} U}{d s^{2}}
$$

In the time domain, we get

$\hat{F}=\frac{5 !}{2 T^{5}} \int_{t-T}^{t}\left(\left(M^{2}-4 \sigma M+\sigma^{2}\right) y(\sigma)-\frac{\alpha}{2} M^{2} \sigma^{2} u(\sigma)\right) d \sigma$,

where $M=T-\sigma$. The choice of the window $T$ results in a trade-off. The larger is $T$, the smaller is the effect of noise but the larger is the error due to truncation. For a precise mathematical foundation on the treatment of the noise through iterated time integrals, see [8], which is based on non-standard analysis, and also [13] which rests on Jacobi polynomials. The closed-loop control applied to the ultra-local model (5) is defined as the so-called intelligent controller

$$
u=-\frac{\hat{F}-y_{d}^{(2)}+\mathfrak{C}(e)}{\alpha}
$$

where $y_{d}$ is the output reference trajectory, $e=y-y_{d}$ is the tracking error, and $\mathfrak{C}(e)=K_{p} e+K_{d} \dot{e}$ is a PD controller. Combining (5) and (10), it yields the following closed-loop error dynamics

$$
\ddot{e}+K_{d} \dot{e}+K_{p} e=e_{F}=F-\hat{F} .
$$

If the estimate $\hat{F}$ is good, the error $e_{F} \simeq 0$ is small and choosing the gains such that $K_{p}>0, K_{d}>0$ guarantees a good tracking of $y_{d}$.

\section{B. Cascaded-model-free approach for the quadrotor}

Problem statement: We want to ensure that the quadrotor tracks the desired time-varying position trajectory $\left(x_{d}, y_{d}, z_{d}\right)$ and yaw angle $\psi_{d}$ without precise information of the physical parameters and forces (for instance, the mass, the inertias and the aerodynamic forces) and despite external disturbances. We propose a cascaded model-free setting of the quadrotor based on a minimal nominal dynamics (see Equations (12), (14), (19a)-(19c)).

Inspired by the quadrotor dynamics structure, we divide the control in two stages (see Fig. 2): the outer loop with slow dynamics which controls the position, and the inner loop with fast dynamics which controls the attitude. From the quadrotor structure, we observe that the position dynamics depends on the attitude dynamics.

\section{Outer-loop Position control}

The model-free setting of the vertical $z$-dynamics (1c) is given by

$$
\ddot{z}=F_{z}+\alpha_{z} u_{1}
$$

where $F_{z}$ represents the neglected dynamics and the external disturbances, and $\alpha_{z}$ is a constant parameter. To estimate the time-varying $F_{z}$ over a time interval of length $T$, we use Equation (9). We set the control law for the thrust $u_{1}$ as:

$$
u_{1}=\frac{1}{\alpha_{z}}\left(-\hat{F}_{z}+\ddot{z}_{d}-K_{p z} e_{z}-K_{d z} \dot{e}_{z}\right)
$$

where $e_{z}=z-z_{d}$ and $\dot{e}_{z}=\dot{z}-\dot{z}_{d}$ are the tracking errors of the vertical position and velocity respectively. To operate safely the quadrotor requires a positive thrust $u_{1}>0$.

The model-free setting of the $x y$-dynamics (1a)-(1b) is given by

$$
\left(\begin{array}{l}
\ddot{x} \\
\ddot{y}
\end{array}\right)=\left(\begin{array}{l}
F_{x} \\
F_{y}
\end{array}\right)+\alpha_{x y} R_{\psi}\left(\begin{array}{c}
\sin \phi \\
\sin \theta \cos \phi
\end{array}\right) u_{1} .
$$

where $\alpha_{x y}=\left(\alpha_{x}, \alpha_{y}\right)^{T}$ are constants, and $F_{x}$ and $F_{y}$ are the neglected dynamics and disturbances for $x$ and $y$ respectively. The rotation matrix $R_{\psi} \in S O(2)$ is given by

$$
R_{\psi}=\left(\begin{array}{cc}
\sin \psi & \cos \psi \\
-\cos \psi & \sin \psi
\end{array}\right), R_{\psi}^{-1}=R_{\psi}^{T}, \quad\left\|R_{\psi}\right\| \leqslant 1 .
$$

Here, $\|\cdot\|$ denotes the Euclidean norm.

Setting a new virtual input $\left(v_{x}, v_{y}\right)^{T}$ defined as:

$$
\left(\begin{array}{l}
\ddot{x} \\
\ddot{y}
\end{array}\right)=\left(\begin{array}{l}
v_{x} \\
v_{y}
\end{array}\right)=R_{\psi}\left(\begin{array}{c}
\sin \phi_{d} \\
\sin \theta_{d} \cos \phi_{d}
\end{array}\right) u_{1},
$$




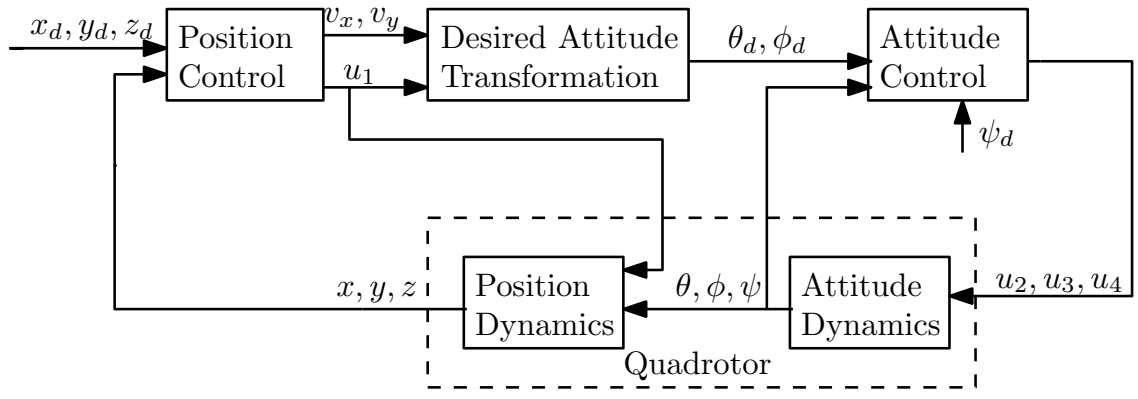

Fig. 2. Cascaded control overview (The subscript $d$ denotes desired trajectory.)

we obtain

$$
\begin{aligned}
& v_{x}=\frac{1}{\alpha_{x}}\left(-\hat{F}_{x}+\ddot{x}_{d}-K_{p x} e_{x}-K_{d x} \dot{e}_{x}\right), \\
& v_{y}=\frac{1}{\alpha_{y}}\left(-\hat{F}_{y}+\ddot{y}_{d}-K_{p y} e_{y}-K_{d y} \dot{e}_{y}\right) .
\end{aligned}
$$

where $e_{x}=x-x_{d}$ and $e_{y}=y-y_{d}$ are the tracking errors for $x$ and $y$ respectively. From there, we can deduce the reference trajectories $\theta_{d}$ and $\phi_{d}$ for the attitude dynamics by expressing the virtual input measures $v_{x}$ and $v_{y}$ in the body frame where $\psi=0$ (no yaw dependence) by:

$$
\left(\begin{array}{c}
\sin \phi_{d} \\
\sin \theta_{d} \cos \phi_{d}
\end{array}\right)=\frac{1}{u_{1}} R_{\psi}^{-1}\left(\begin{array}{l}
v_{x} \\
v_{y}
\end{array}\right) \triangleq\left(\begin{array}{c}
\bar{v}_{x} \\
\bar{v}_{y}
\end{array}\right)
$$

Hence we consider the following desired angles $\phi_{d}$ and $\theta_{d}$ :

$$
\begin{aligned}
\phi_{d} & =\arcsin \left(\bar{v}_{x}\right), \\
\theta_{d} & =\arcsin \left(\frac{\bar{v}_{y}}{\cos \phi_{d}}\right) .
\end{aligned}
$$

\section{Inner-loop Attitude control}

The attitude dynamics (2a)-(2c) are given by the following:

$$
\begin{aligned}
& \ddot{\phi}=F_{\phi}+\alpha_{\phi} u_{2}, \\
& \ddot{\theta}=F_{\theta}+\alpha_{\theta} u_{3}, \\
& \ddot{\psi}=F_{\psi}+\alpha_{\psi} u_{4}
\end{aligned}
$$

The attitude control performance is crucial since it is directly related to the actuators efficiency. After deducing the desired attitude $\phi_{d}, \theta_{d}$ from the slow outer loop (18a)-(18b), the fast inner loop is stabilized by an MFC controller as

$$
\begin{aligned}
& u_{2}=\frac{1}{\alpha_{\phi}}\left(-\hat{F}_{\phi}+\ddot{\phi}_{d}-K_{p \phi} e_{\phi}-K_{d \phi} \dot{e}_{\phi}\right), \\
& u_{3}=\frac{1}{\alpha_{\theta}}\left(-\hat{F}_{\theta}+\ddot{\theta}_{d}-K_{p \theta} e_{\theta}-K_{d \theta} \dot{e}_{\theta}\right),
\end{aligned}
$$

where $e_{\phi}=\phi-\phi_{d}, e_{\theta}=\theta-\theta_{d}, \dot{e}_{\phi}=\dot{\phi}-\dot{\phi}_{d}, \dot{e}_{\theta}=\dot{\theta}-\dot{\theta}_{d}$ are the tracking errors of the angular positions and estimated angular velocities respectively. Similarly, for the yaw angle, we apply the Model-Free control as

$$
u_{4}=\frac{1}{\alpha_{\psi}}\left(-\hat{F}_{\psi}+\ddot{\psi}_{d}-K_{p \psi} e_{\psi}-K_{d \psi} \dot{e}_{\psi}\right) .
$$

\section{AgRessive trajeCtORY TRACKING}

In this section, we give the predefined aggressive trajectory that the quadrotor will follow. In most cases, the trajectory is a straight line or circular orbit. We choose the B-spline curves (the definition of a B-Spline curve can be found in the Appendix) because they are always contained in the convex hull of their control polygon: the basis functions are positive and sum up to one (partition of unity), and have a local support [12]. By increasing the degree of the B-spline curve and/or by inserting extra knots, the distance between the control polygon and the B-spline curve can be reduced.

We consider here an aggressive motion $\left[x_{d}(t), y_{d}(t), z_{d}(t), \psi_{d}(t)\right] \quad: \quad\left[\begin{array}{ll}t_{0} & t_{f}\end{array}\right] \rightarrow \mathbb{R}^{3} \times S O(2)$ defined as a B-spline curve:

$$
\begin{aligned}
& x_{d}(t)=\sum_{j=1}^{10} a_{x j} B_{j, d}, \\
& y_{d}(t)=\sum_{j=1}^{10} a_{y j} B_{j, d}, \\
& z_{d}(t)=\sum_{j=1}^{10} a_{z j} B_{j, d},
\end{aligned}
$$

with degree $d=4$, knot vector $t=$ $\{0,0,0,0,0,2.5,5,7.5,10,12.5,15,15,15,15,15\}, \quad$ and control point vectors $\boldsymbol{A}_{\boldsymbol{x}}=\left(a_{x j}\right)_{x j=1}^{10}, \boldsymbol{A}_{\boldsymbol{y}}=\left(a_{y j}\right)_{y j=1}^{10}$ and $\boldsymbol{A}_{\boldsymbol{z}}=\left(a_{z j}\right)_{z j=1}^{10}$ for $x_{d}(t), y_{d}(t)$ and $z_{d}(t)$ respectively.

We specialize the reference angle $\psi_{d}$ to a sigmoid between two constant angles a situation frequently needed in practice.

$$
\psi_{d}(t)=\frac{\Psi_{f}-\Psi_{i}}{2}\left(1+\tanh \left(\gamma\left(t-t_{m}\right)\right)\right)+\Psi_{i}
$$

where $\Psi_{i}=0 \mathrm{rad}$ is the initial $\psi$-angle and $\Psi_{f}=1.5 \mathrm{rad}$ is the final $\psi$-angle of the quadrotor; $\gamma=0.4$ is the slope parameter of the sigmoid and $t_{m}=7$.

\section{Simulation Results}

To demonstrate the proposed control we consider a realistic quadrotor simulator where we use the quadrotor model defined by the equations (1a)-(1c), (2a)-(2c) and (3) presented in Section II. Similar to the usual case in practice, the controller runs at $20 \mathrm{~Hz}$, and the sensor data is updated 


\begin{tabular}{|c|c|c|c|c|c|}
\hline $\mathrm{P}$ & $\mathrm{V}$ & $\mathrm{P}$ & $\mathrm{V}$ & $\mathrm{P}$ & $\mathrm{V}$ \\
\hline$K_{p x}$ & 3 & $K_{d x}$ & 2 & $\alpha_{x}$ & 1 \\
$K_{p y}$ & 3 & $K_{d y}$ & 2 & $\alpha_{y}$ & 1 \\
$K_{p z}$ & 10 & $K_{d z}$ & 15 & $\alpha_{z}$ & 2 \\
$K_{p \phi}$ & 2 & $K_{d \phi}$ & 0.5 & $\alpha_{\phi}$ & 1 \\
$K_{p \theta}$ & 1 & $K_{d \theta}$ & 0.5 & $\alpha_{\theta}$ & 1 \\
$K_{p \psi}$ & 3 & $K_{d \psi}$ & 2.5 & $\alpha_{\psi}$ & 9 \\
\hline
\end{tabular}

TABLE II

The control gains (P: Parameter, V:VAlue).

at $100 \mathrm{~Hz}$. The aim here is to track the positions $x_{d}, y_{d}$ and $z_{d}$, and the yaw angle $\psi_{d}$. As initial positions and initial angles, we take $\left(x_{0}, y_{0}, z_{0}\right)=(-1.8,1,0)[\mathrm{m}]$ and $\left(\phi_{0}, \theta_{0}, \psi_{0}\right)=(0,0,0)[\mathrm{rad}]$ respectively. The initial linear velocities and initial angular velocities are zero. The control point vectors for the reference trajectories are the following:

$$
\begin{aligned}
& \boldsymbol{A}_{\boldsymbol{x}}=\{-2,-1,0,2,3,3.5,3.5,5,6.5,7.5\}, \\
& \boldsymbol{A}_{\boldsymbol{y}}=\{1.2,2.5,3.3,1.8,1.5,2.5,4,4,4,4\}, \\
& \boldsymbol{A}_{\boldsymbol{z}}=\{0,0.2,0.5,2.5,3.5,3.9,4.7,6.5,5.7,3.5\} .
\end{aligned}
$$

Our trajectory is parametrized to avoid known static obstacles is a situation frequently needed in practice. In the nominal case (without disturbances), we observe in Fig. 3 (trajectory tracking), Fig. 4 (control inputs) and Fig. 5 (unknown dynamics estimation) satisfactory results when the position and the yaw angle change significantly. The 3D tracking is plotted in Fig. 6. The control gains that we consider are given in Table II. The values for $\alpha_{i}$ are chosen from a large possible range each dependant on the corresponding state dynamics.

\section{A. Scenario 1: Unknown measurement noise}

For this scenario, we include the presence of the measurement noise $b(t) \sim \mathcal{N}\left(0, \sigma^{2}\right)$ as an additive white Gaussian noise with zero mean and standard deviation $\sigma=0.15$ in the four measured outputs $x, y, z$ and $\psi$. The noisy measured outputs are set as:

$$
\begin{array}{ll}
\hat{x}=x+b, & \hat{y}=y+b, \\
\hat{z}=z+b, & \hat{\psi}=\psi+b .
\end{array}
$$

Fig. 7 shows the noisy sensor measurements (green line) and the tracking trajectory of the quadrotor (blue line) that follows the desired trajectory (red line). From the result in Fig. 7 (trajectory tracking) and Fig. 8 (control inputs), it is apparent that the control approach is robust to the sensor disturbances without previous knowledge of the noise.

\section{B. Scenario 2: Unknown time-varying wind disturbance}

In this scenario, we investigate the quadrotor tracking in presence of wind disturbance that is not constant and is not assumed to be known. The high varying wind disturbances used in the simulations (displayed in Fig.11) are represented by a sum of sinusoidal waves:

$$
w(t)=1.5 \mu_{31}+\mu_{7}+0.5 \mu_{2}+0.015 \mu_{11}+0.15 b(t)
$$

where $\mu_{p}(t)=\sin \left(\frac{t \pi}{p}\right)$ and $b(t)$ is the random Gaussian noise. We add this disturbance in the simulation model as an additive force disturbance such that

$$
m \ddot{x}=(\sin \psi \sin \phi+\cos \psi \sin \theta \cos \phi) u_{1}-A_{x} \dot{x}+w(t),
$$

$m \ddot{y}=(-\cos \psi \sin \phi+\sin \psi \sin \theta \cos \phi) u_{1}-A_{y} \dot{y}+w(t)$,

$$
m \ddot{z}=-m g+(\cos \theta \cos \phi) u_{1}-A_{z} \dot{z}+w(t) .
$$

We observe the unknowns $F_{i}$ for $i=x, y, z, \psi$ in the nominal case (see Fig. 5) and in the presence of wind disturbance (see Fig. 10). We show that the precise estimation of the unknown $F_{i}$ for $i=x, y, z, \psi$ leads us to a robust control performance. The trajectory tracked by the quadrotor in presence of time-varying wind disturbance depicted in Fig. 9 is almost identical to the nominal case.

\section{CONCLUSION}

This methodology offers a cascaded model-free control design of a quadrotor. It stays robust, despite unknown disturbances. We have shown that a complex trajectory tracking on realistic scenarios is feasible. In the future works, we shall test our approach on an experimental platform. Considering the low complexity and the good performances of the proposed control, it sheds new lights on a possible commercial off-the-shelf solution. A complete stability analysis of the proposed system (quadrotor and cascaded modelfree controller) is in final stages and will be presented in a forthcoming publication.

\section{APPENDIX}

Definition 1: (B-splines [12])

Let the degree $d$ be a nonnegative integer and let the knot vector $\boldsymbol{t}=\left(t_{j}\right)$, be a non-decreasing sequence of real numbers of at least $d+2$. The $j$ th B-spline of degree $d$ with knots $t$ is defined by:

$B_{j, k, \boldsymbol{t}}(x)=\frac{x-t_{j}}{t_{j+d}-t_{j}} B_{j, d-1, \boldsymbol{t}}+\frac{t_{j+k}-x}{t_{j+1+d}-t_{j+1}} B_{j+1, d-1, \boldsymbol{t}}$

for all real numbers $x$, with

$$
B_{j, 0, \boldsymbol{t}}(x)= \begin{cases}1, & \text { if } t_{j} \leqslant x<t_{j+1} \\ 0, & \text { otherwise. }\end{cases}
$$

Definition 2: (B-spline curves). Let a knot vector for a total of $n$ B-splines be defined as:

$$
\boldsymbol{t}=\left(t_{j}\right)_{j=1}^{m=n+d+1}=[\underbrace{0, \ldots, 0}_{d+1}, t_{d+1}, \ldots, t_{m-d-1}, \underbrace{1, \ldots, 1}_{d+1}]
$$

The linear space of all linear combinations of these B-splines is the spline space $\mathbb{S}_{d, \boldsymbol{t}}$ defined by

$$
\mathbb{S}_{d, \boldsymbol{t}}=\left\{\sum_{j=1}^{n} c_{j} B_{j, d} \mid c_{j} \in \mathbb{R} \text { for } 1 \leqslant j \leqslant n\right\}
$$



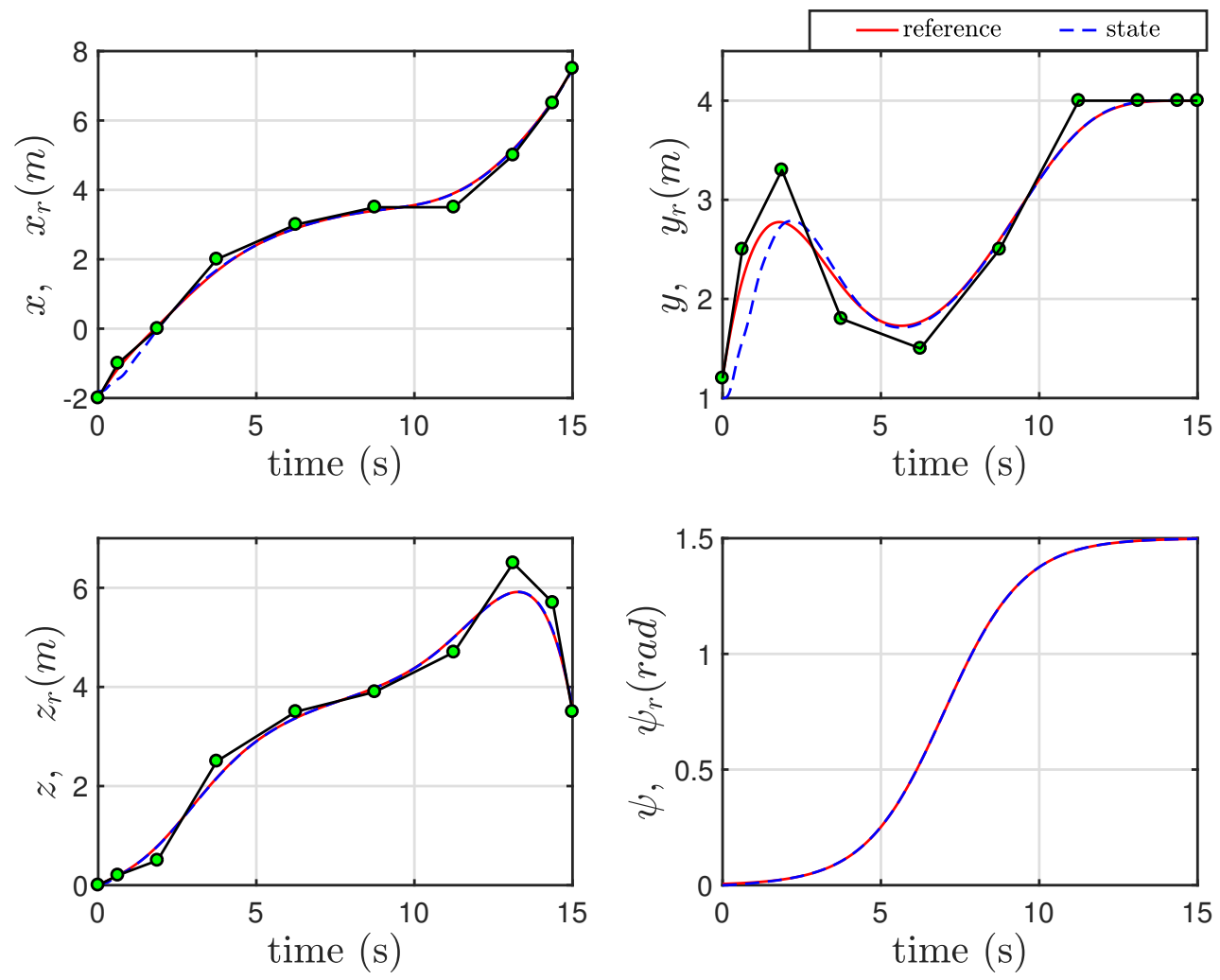

Fig. 3. Reference trajectories and model-free tracking trajectories of the four outputs $x, y, z$ and $\psi$ in the nominal case
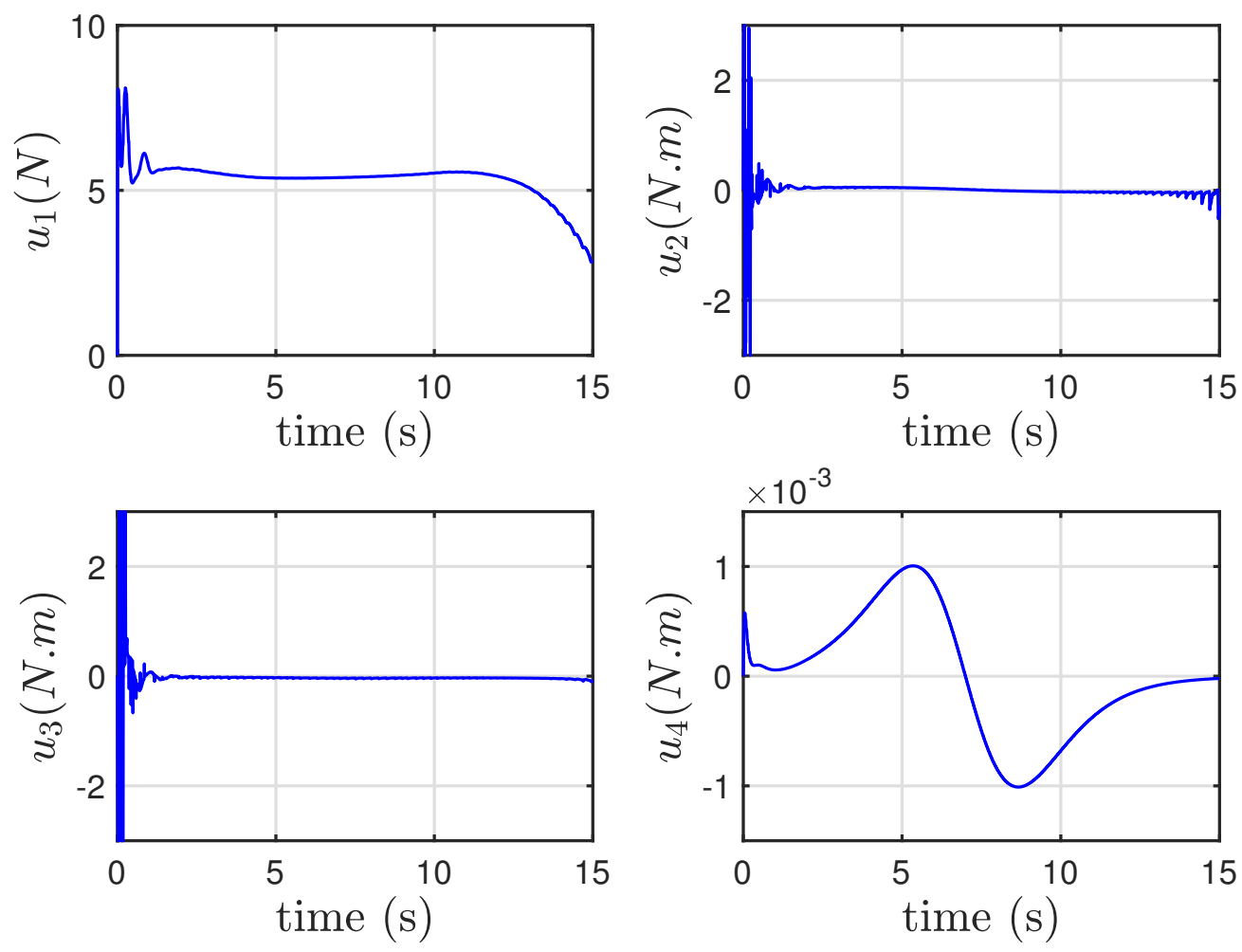

Fig. 4. Control inputs in the nominal case 

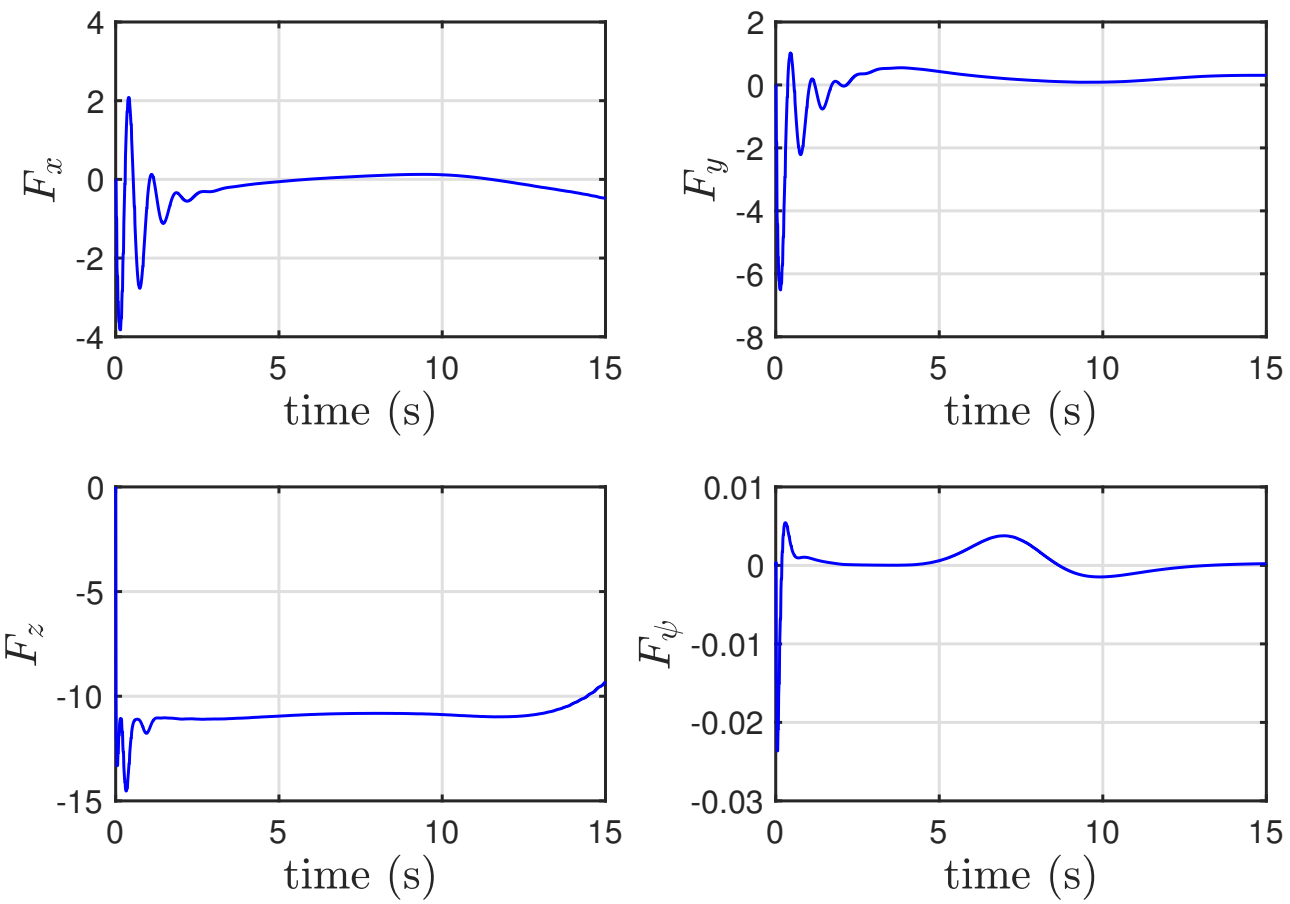

Fig. 5. Estimation of the unknowns $F_{i}$ for $i=x, y, z, \psi$ in the nominal case

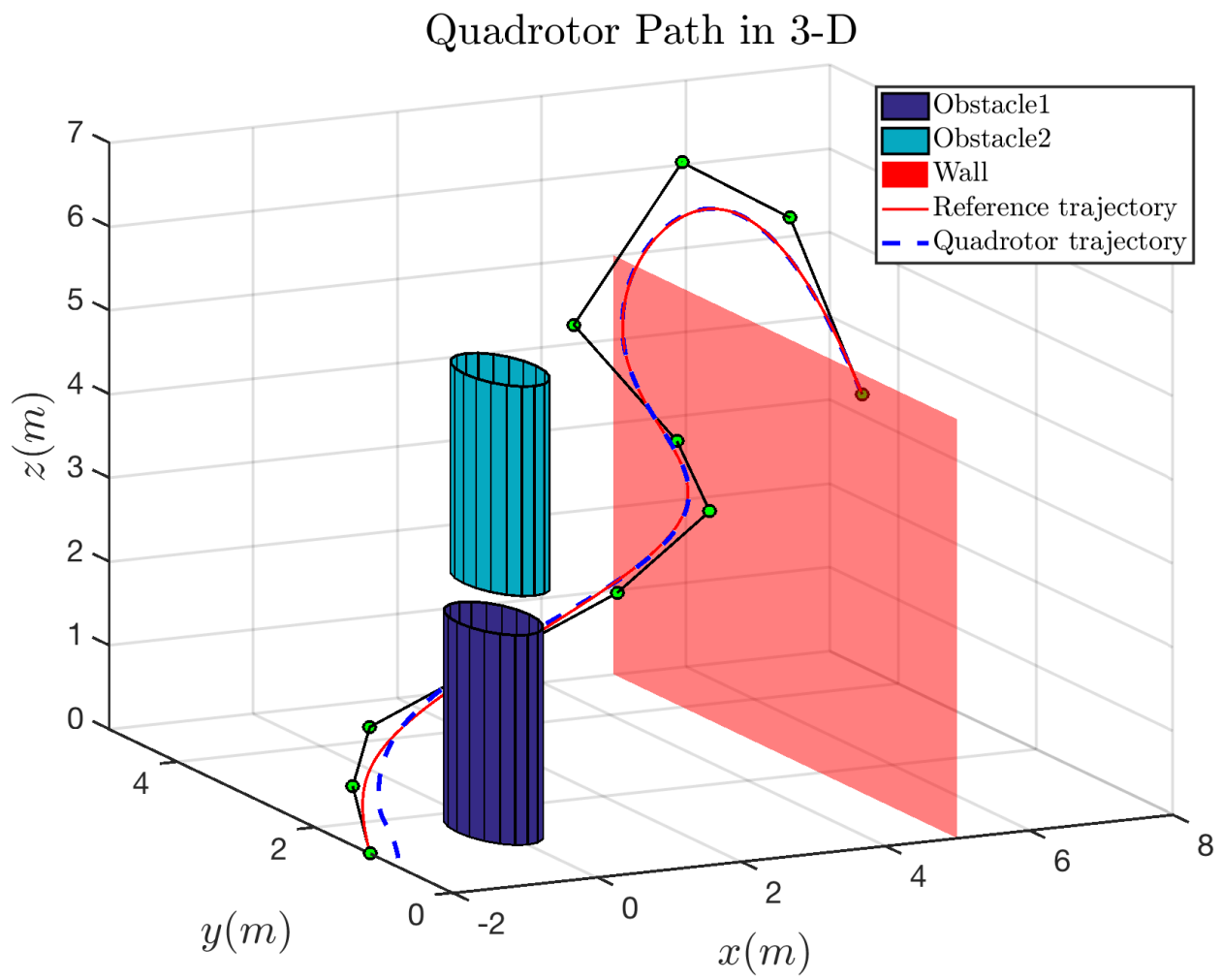

Fig. 6. 3-D Position tracking of the B-spline path in the nominal case. (Objects in the simulation are given just as potential obstacles, but no obstacle avoidance algorithm is yet used.) 

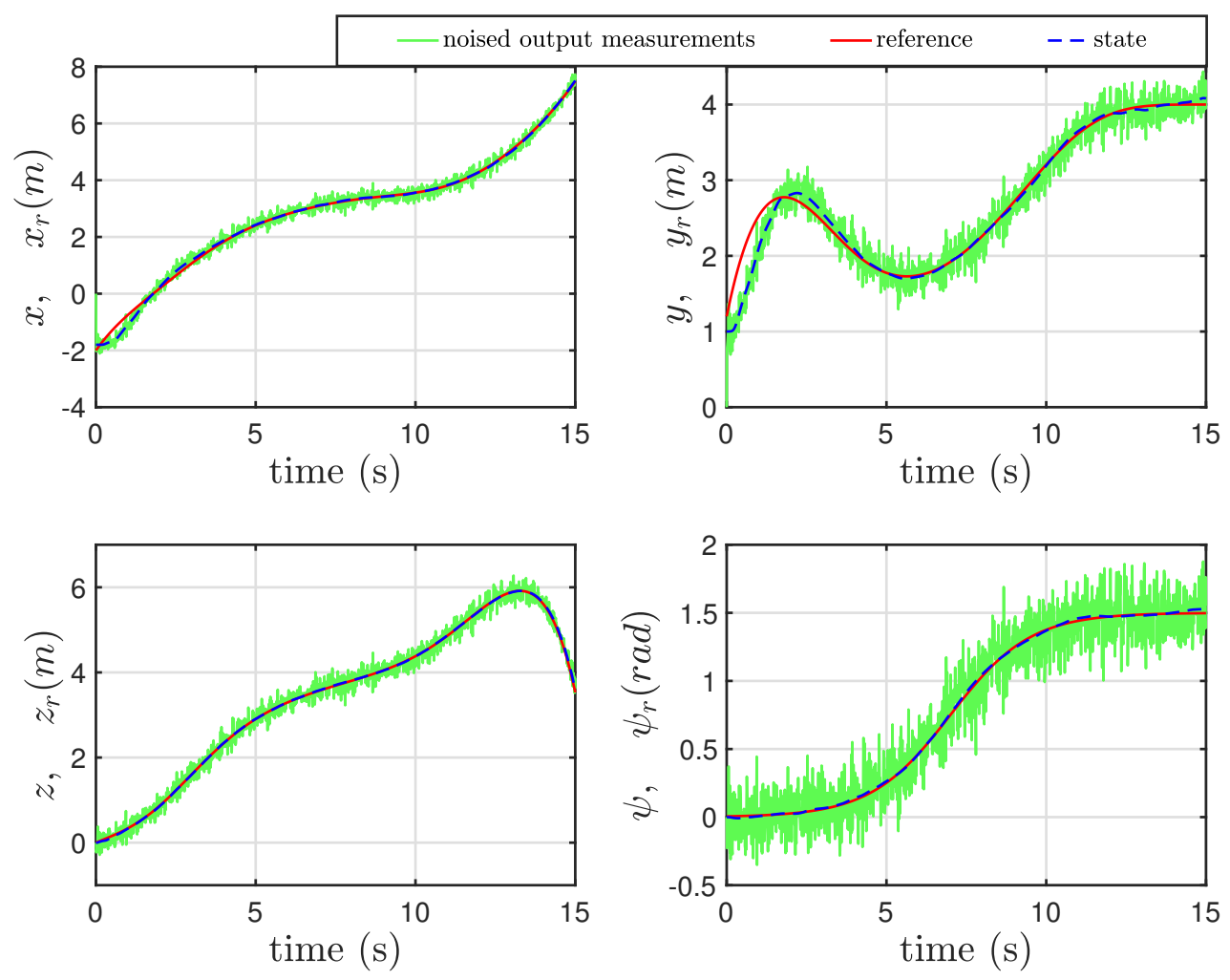

Fig. 7. Tracking trajectories in presence of noise (noisy measurements and real state position)
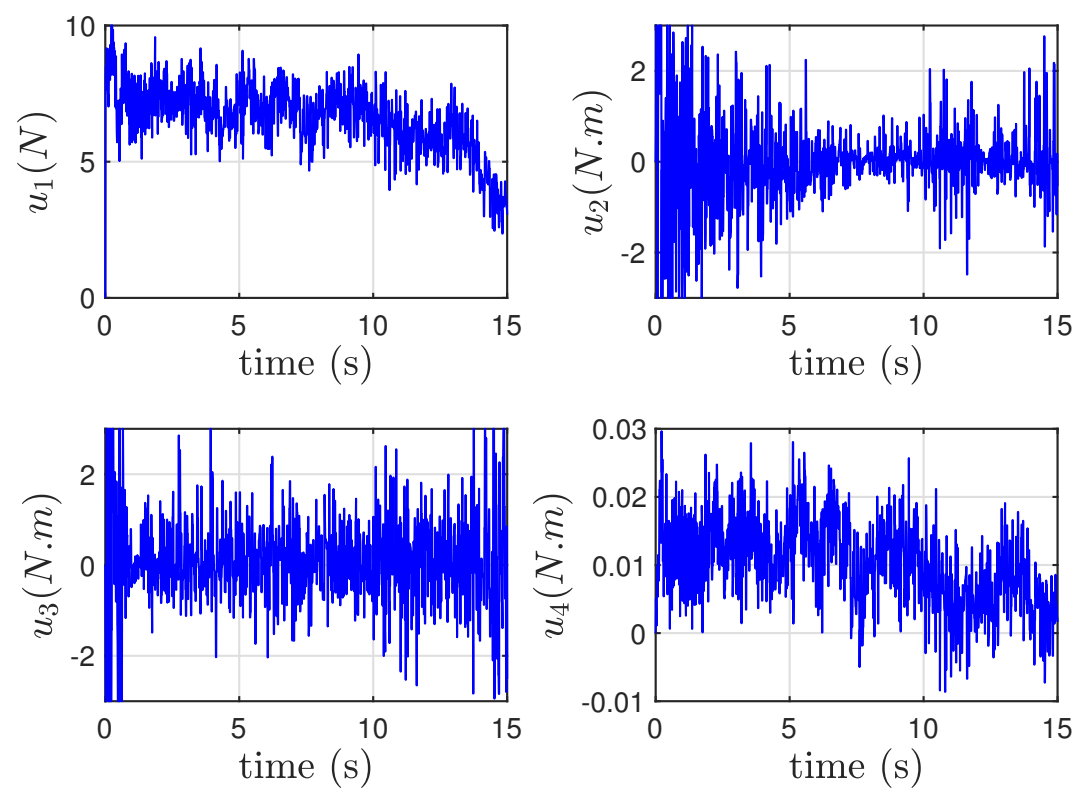

Fig. 8. Control inputs in presence of noise 

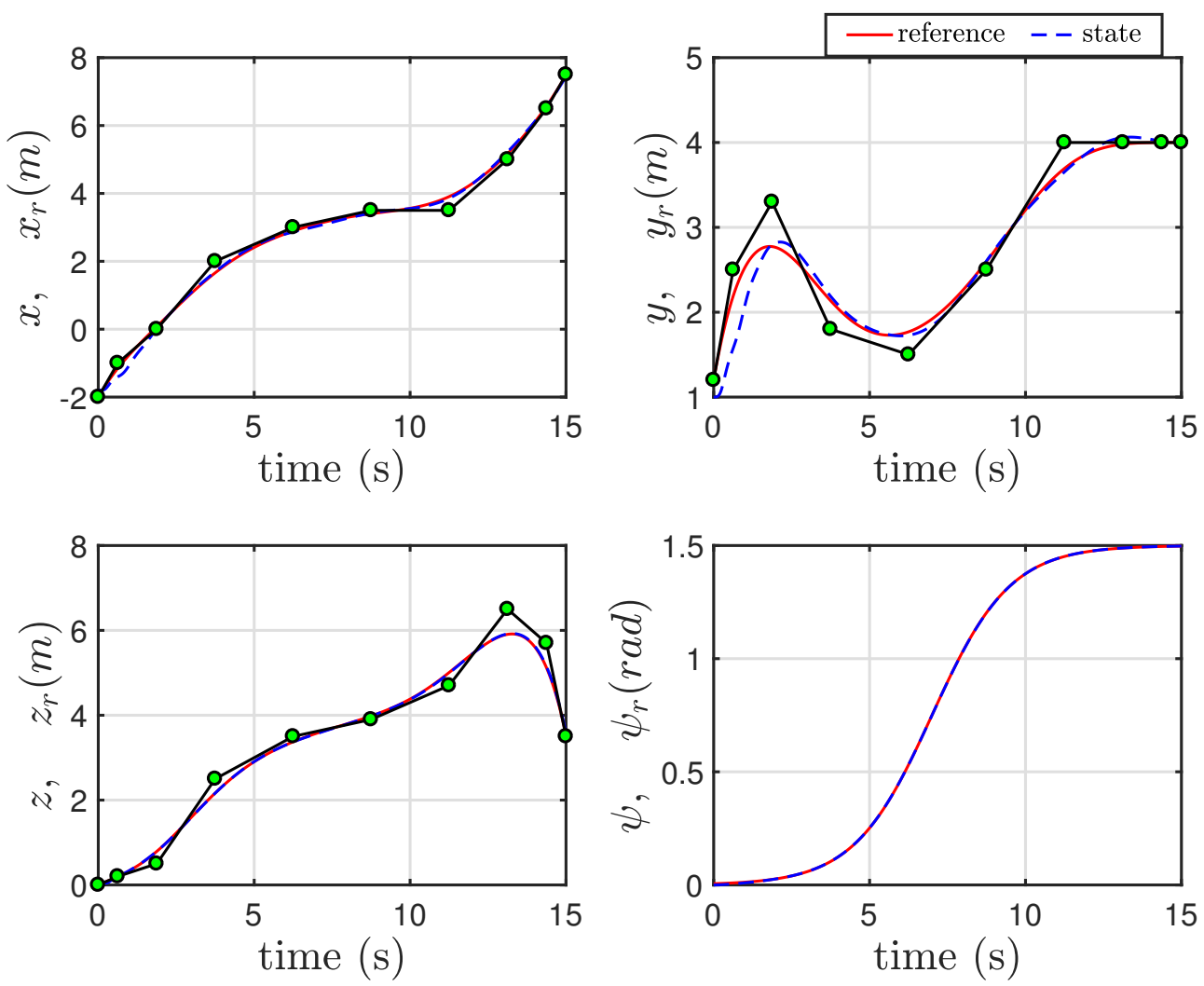

Fig. 9. Tracking outputs in presence of wind disturbance
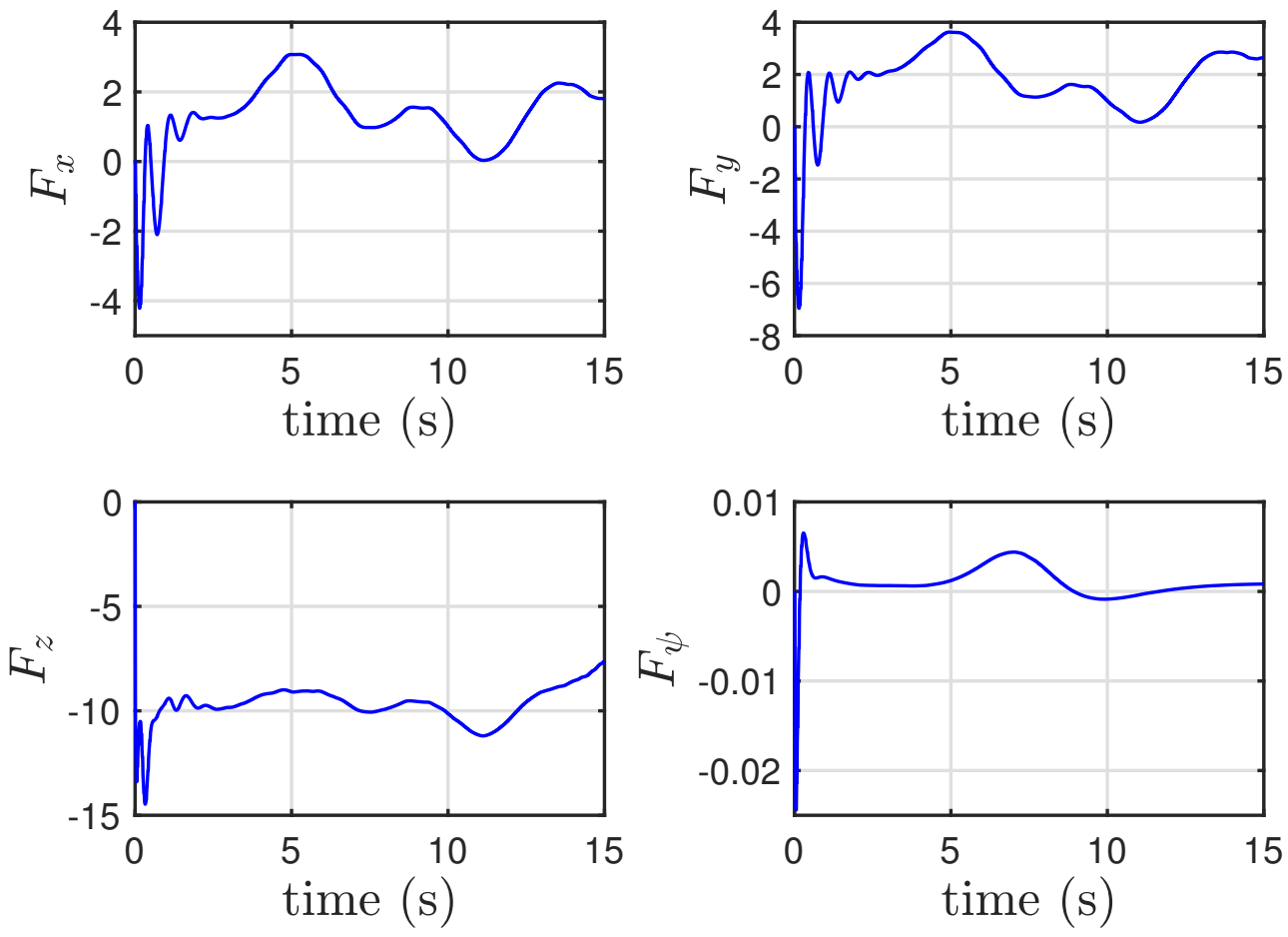

Fig. 10. Estimation of the unknowns $F_{i}$ for $i=x, y, z, \psi$ in presence of wind disturbance 


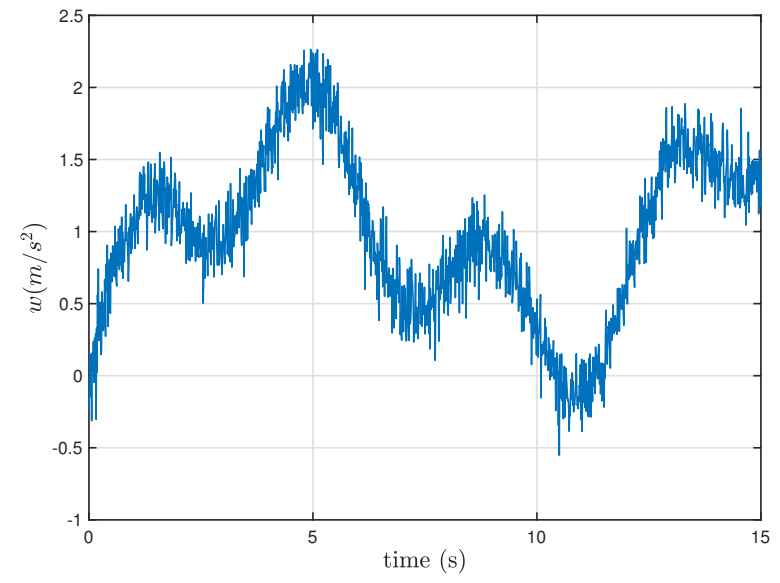

Fig. 11. Wind disturbance

An element $f=\sum_{j=1}^{n} c_{j} B_{j, d}$ of $\mathbb{S}_{d, \boldsymbol{t}}$ is called a B-spline curve or a spline function, of degree $d$ with knots $\boldsymbol{t}$, and $\left(c_{j}\right)_{j=1}^{n}$ are called the control points of the B-spline curve.

\section{REFERENCES}

[1] H. Abouaïssa, M. Fliess, and C. Join. On ramp metering: towards a better understanding of ALINEA via model-free control. International Journal of Control, 90(5):1018-1026, 2017.

[2] K. Alexis, G. Nikolakopoulos, and A. Tzes. Model predictive quadrotor control : attitude, altitude. IET Control Theory and Applications, 6(June 2011):1812-1827, 2012.

[3] S. Bouabdallah, A. Noth, and R. Siegwart. PID vs LQ control techniques applied to an indoor micro quadrotor. In IEEE/RSJ International Conference on Intelligent Robots and Systems (IROS), volume 3, pages 2451-2456, 2004.

[4] S. Bouabdallah and R. Siegwart. Backstepping and sliding-mode techniques applied to an indoor micro Quadrotor. In IEEE International Conference on Robotics and Automation (ICRA), pages 2247-2252, 2005.

[5] N. Cao and A. F. Lynch. Inner-Outer Loop Control for Quadrotor UAVs with Input and State Constraints. IEEE Transactions on Control Systems Technology, 24(5):1797-1804, 2016.

[6] B. D'Andréa-Novel, M. Fliess, C. Join, H. Mounier, and B. Steux. A mathematical explanation via intelligent PID controllers of the strange ubiquity of PIDs. In 18th Mediterranean Conference on Control and Automation (MED), pages 395-400, 2010.

[7] M. Doublet, C. Join, and F. Hamelin. Model-free control for unknown delayed systems. In Conference on Control and Fault-Tolerant Systems, SysTol, 2016.

[8] M. Fliess. Analyse non standard du bruit. Comptes Rendus Mathematique, 342(10):797-802, 2006.

[9] M. Fliess and C. Join. Model-free control. International Journal of Control, 86(12):2228-2252, 2013

[10] M. Fliess and H. Sira-Ramirez. Closed-loop parametric identification for continuous-time linear systems via new algebraic techniques. In Identification of Continuous-time Models from Sampled Data, pages 362-391. Springer London, 2008.

[11] T. Luukkonen. Modelling and Ccontrol of Quadcopter. Independent research project in applied mathematics, Espoo., 2011.

[12] T. Lyche and K. Morken. Spline Methods. page 36, 2002.

[13] M. Mboup, C. Join, and M. Fliess. Numerical differentiation with annihilators in noisy environment. Numerical Algorithms- Springer Verlag, 4(50):1-27, 2009.

[14] D. Mellinger, N. Michael, and V. Kumar. Trajectory generation and control for precise aggressive maneuvers with quadrotors. Springer Tracts in Advanced Robotics, 79(2009):361-373, 2014.

[15] L. Menhour, B. D'Andrea-Novel, M. Fliess, D. Gruyer, and H. Mounier. An Efficient Model-Free Setting for Longitudinal and Lateral Vehicle Control: Validation Through the Interconnected Pro-
SiVIC/RTMaps Prototyping Platform. IEEE Transactions on Intelligent Transportation Systems, 19(2):pp.461-475, 2017.

[16] P. Polack, B. D'Andréa-Novel, M. Fliess, A. de la Fortelle, and L. Menhour. Finite-Time Stabilization of Longitudinal Control for Autonomous Vehicles via a Model-Free Approach. In IFAC World Congress, 2017.

[17] P. Pounds, R. Mahony, and P. Corke. Modelling and control of a large quadrotor robot. Control Engineering Practice, 18(7):691-699, 2010.

[18] A. Sanchez-Orta, V. Parra-Vega, C. Izaguirre-Espinosa, and O. Garcia. Position-Yaw Tracking of Quadrotors. Journal of Dynamic Systems, Measurement, and Control, 137(6):061011, 2015.

[19] H. Sira-Ramirez. On the linear control of the quad-rotor system. In Proceedings of the 2011 American Control Conference, pages 31783183, 2011.

[20] C. Wang, B. Song, P. Huang, and C. Tang. Trajectory Tracking Control for Quadrotor Robot Subject to Payload Variation and Wind Gust Disturbance. Journal of Intelligent and Robotic Systems: Theory and Applications, 83(2):315-333, 2016.

[21] H. Wang, X. Ye, Y. Tian, G. Zheng, and N. Christov. Model-free-based terminal SMC of quadrotor attitude and position. IEEE Transactions on Aerospace and Electronic Systems, 52(5):2519-2528, 2016.

[22] J. Wang, H. Mounier, S.-I. Niculescu, M.-S. Geamanu, and A. Cela. Event-driven model-free control in motion control with comparisons. IMA Journal of Mathematical Control and Information, 34(4):12551275, 2016.

[23] Y. A. Younes, A. Drak, H. Noura, A. Rabhi, and A. E. Hajjaji. Robust Model-Free Control Applied to a Quadrotor UAV. Journal of Intelligent and Robotic Systems: Theory and Applications, 84(14):37-52, 2016 